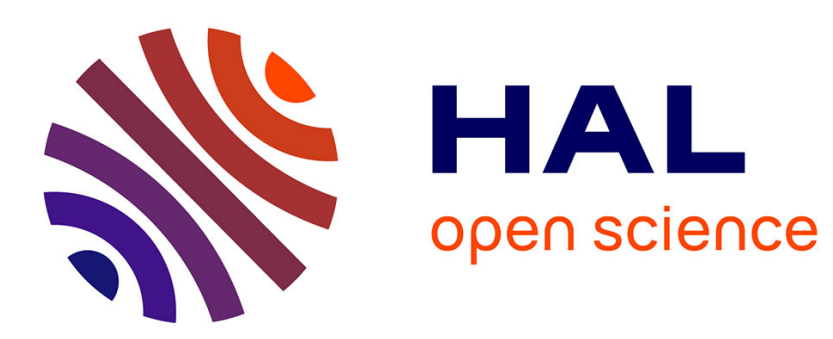

\title{
Derivation of a modified Korteweg-de Vries model for few-optical-cycles soliton propagation from a general Hamiltonian
}

\author{
Houria Triki, Hervé Leblond, Dumitru Mihalache
}

\section{- To cite this version:}

Houria Triki, Hervé Leblond, Dumitru Mihalache. Derivation of a modified Korteweg-de Vries model for few-optical-cycles soliton propagation from a general Hamiltonian. Optics Communications, 2012, 285 (13-14), pp.3179 - 3186. 10.1016/j.optcom.2012.02.045 . hal-03192911

\section{HAL Id: hal-03192911 \\ https://univ-angers.hal.science/hal-03192911}

Submitted on 8 Apr 2021

HAL is a multi-disciplinary open access archive for the deposit and dissemination of scientific research documents, whether they are published or not. The documents may come from teaching and research institutions in France or abroad, or from public or private research centers.
L'archive ouverte pluridisciplinaire HAL, est destinée au dépôt et à la diffusion de documents scientifiques de niveau recherche, publiés ou non, émanant des établissements d'enseignement et de recherche français ou étrangers, des laboratoires publics ou privés. 


\title{
Derivation of a modified Korteweg-de Vries model for few-optical-cycles soliton propagation from a general Hamiltonian
}

\author{
H. Triki ${ }^{\mathrm{b}}$, H. Leblond ${ }^{\mathrm{a}, *}$, D. Mihalache ${ }^{\mathrm{a}, \mathrm{c}, \mathrm{d}}$ \\ a LUNAM Université, Université d'Angers, Laboratoire de Photonique d'Angers, EA 4464, 2 Bd. Lavoisier, 49045 Angers Cedex 01, France \\ ${ }^{b}$ Radiation Physics Laboratory, Department of Physics, Faculty of Sciences, Badji Mokhtar University, P. O. Box 12, 23000 Annaba, Algeria \\ ${ }^{\mathrm{c}}$ Horia Hulubei National Institute for Physics and Nuclear Engineering (IFIN-HH), 30 Reactorului, Magurele-Bucharest, 077125, Romania \\ d Academy of Romanian Scientists, 54 Splaiul Independentei, Bucharest 050094, Romania
}

\section{A R T I C L E I N F O}

\section{Article history:}

Received 11 October 2011

Received in revised form 18 February 2012

Accepted 20 February 2012

Available online 3 March 2012

\section{Keywords:}

Few-cycle pulses

Few-cycle solitons

Modified Korteweg-de Vries equation

mKdV equation

\begin{abstract}
A B S T R A C T
Propagation of few-cycles optical pulses in a centrosymmetric nonlinear optical Kerr (cubic) type material described by a general Hamiltonian of multilevel atoms is considered. Assuming that all transition frequencies of the nonlinear medium are well above the typical wave frequency, we use a long-wave approximation to derive an approximate evolution model of modified Korteweg-de Vries type. The model derived by rigorous application of the reductive perturbation formalism allows one the adequate description of propagation of ultrashort (few-cycles long) solitons.
\end{abstract}

(c) 2012 Elsevier B.V. All rights reserved.

\section{Introduction}

Since 1999, few-optical cycle pulses [1-4] with durations of only a few periods of the optical radiation have become the primary components in many important problems of dynamics of nonlinear optical waves. In particular, these ultrashort (femtosecond) pulses [5,6] find applications in a wide variety of research areas, such as lightmatter interactions at high field intensities, high-order harmonic generation, extreme nonlinear optics [7], and attosecond physics $[8,9]$. Notably, the theoretical modeling used to correctly describe the dynamics of such pulses in nonlinear optical media has also been developed in parallel to these incentive experimental studies. It should be said that, the search for new ideas or even new mathematical concepts is of great interest as it is helpful to better understand the ultrashort pulse propagation in nonlinear optical media and the formation of robust few-optical-cycle solitons.

The continuing experimental progress in the study of wave dynamics of few-cycle pulses (FCPs) in nonlinear optical media has paved the way for the development of new theoretical approaches to model their propagation in a lot of physical settings. Three classes of main dynamical models for FCPs have been put forward in the past years: (i) the quantum approach [10-14], (ii) the refinements within the framework of slowly varying envelope approximation (SVEA) of the nonlinear Schrödinger-type envelope equations [15-24], and

\footnotetext{
* Corresponding author. Tel.: + 332417354 31; fax: + 33241735216 E-mail address: herve.leblond@univ-angers.fr (H. Leblond).
}

(iii) the non-SVEA models [25-43]. The propagation of FCPs in Kerr media can be described beyond the SVEA by using the modified Korteweg-de Vries (mKdV) [31-33], sine-Gordon (sG) [34-36], or $\mathrm{mKdV}-\mathrm{sG}$ equations [37-41]. The $\mathrm{mKdV}$ and $\mathrm{sG}$ equations are completely integrable by means of the inverse scattering transform method $[44,45]$, whereas the $\mathrm{mKdV}-\mathrm{sG}$ equation is completely integrable only if some condition between its coefficients is satisfied $[46,47]$.

Other relevant works on few-cycle pulses deal with propagation and interaction of extremely short electromagnetic pulses in quadratic nonlinear media [48-51], the study of few-cycle light bullets created by femtosecond filaments [52], the investigation of ultrashort spatiotemporal optical solitons in quadratic nonlinear media [53], the ultrashort spatiotemporal optical pulse propagation in cubic (Kerr-like) media without the use of the slowly varying envelope approximation $[54,55]$, the possibility of generating few-cycle dissipative optical solitons [56,57], generation of unipolar pulses from nonunipolar optical pulses in a quadratic nonlinear medium [58], and the existence of guided optical solitons of femtosecond duration and nanoscopic mode area, that is, femtosecond nanometer-sized optical solitons [59].

We also mention recent studies of ultrafast pulse propagation in mode-locked laser cavities in the few femtosecond pulse regime and the derivation of a master mode-locking equation for ultrashort pulses [60]. A relevant recent theoretical work presents a class of few-cycle elliptically polarized solitary waves in isotropic Kerr media, propose a method of producing multisolitons with different polarization states, and study their binary-collision dynamics [61]. Robust circularly polarized few-optical-cycle solitons in Kerr media 
in both long-wave and short-wave approximation regimes were also studied in recent works [62-64].

So far, most of theoretical investigations concern only FCPs propagating in a nonlinear optical medium which is described by a Hamiltonian related to two-level atoms. However, to the best of our knowledge, studies based on very general Hamiltonians describing the wave dynamics of such ultrashort pulses have not yet been reported. It is, nevertheless, an interesting and potentially useful description if FCPs can form and can be robust when we consider this more general physical setting. The aim of this paper is to investigate such a multilevel system in the framework of the reductive perturbation method (multiscale analysis). This fact allows one to extend the existing studies to a more general physical situation. Thus in this work we give a detailed mathematical derivation of the modified Korteweg-de Vries equation for a general Hamiltonian. We assume that the absorption spectrum of the nonlinear medium does not extend below some cutoff frequency, and that the typical frequency of the FCP is much less than the latter. In other words, we assume that the transparency range of the medium is very large, and consider only the frequencies located in the ultraviolet spectral domain and further. The effect of the infrared transitions, which yield a sineGordon model in the case of two-level atoms, will be considered in a further study.

The present study can be considered from two different points of view: (i) a nonlinear cubic medium which has no transition in the infrared is actually described, and (ii) the most general medium containing two kinds of transitions, both in the infrared and in the ultraviolet domains, is expected to be described by a more general modified Korteweg-de Vries-sine-Gordon equation. However, we give here the detailed mathematical derivation for the modified Korteweg-de Vries part of the most general model.

This paper is organized as follows. In the next section we present in detail a derivation of the modified-Korteweg-de Vries equation as a rigorous formal asymptotics of the Maxwell-Bloch equations for the most general Hamiltonian for multilevel atoms. This evolution equation describes the propagation of ultrashort (a few femtosecond long) optical solitons in the so-called long-wave regime. In Section 3 we analyze both analytically and numerically the modified Korteweg-de Vries breather, which is the prototype of fewoptical-cycle solitons in such cubic nonlinear optical media. Finally, in Section 4 we present our conclusions.

\section{The derivation of a modified Korteweg-de Vries model from a general Hamiltonian}

We consider a set of multilevel atoms described by a very general Hamiltonian:

$H_{0}=\hbar\left(\begin{array}{cccc}\omega_{1} & 0 & \cdots & 0 \\ 0 & \omega_{2} & \cdots & \cdots \\ \cdots & \cdots & \cdots & \cdots \\ 0 & \cdots & \cdots & \omega_{N}\end{array}\right)$

The evolution of the density matrix $\rho$ is governed by the Schrödingervon Neumann equation

$i \hbar \partial_{t} \rho=[H, \rho]$,

in which the total Hamiltonian

$H=H_{0}-\vec{\mu} \cdot \vec{E}$

includes a term accounting for the coupling between the electric field $\vec{E}$ and the atoms through a dipolar momentum operator $\vec{\mu}$.
The evolution of the electric field is governed by Maxwell-Helmholtz wave equation

$\partial_{z}^{2} \vec{E}=\frac{1}{c^{2}} \partial_{t}^{2}\left(\vec{E}+\frac{1}{\varepsilon_{0}} \vec{P}\right)$

in which the polarization density $\vec{P}$ expresses as

$\vec{P}=N \operatorname{Tr}(\rho \vec{\mu})$

We consider a cubic (Kerr) nonlinearity, i.e. we assume that the material is centrosymmetric, so that the second order susceptibility $\chi^{(2)}$ vanishes. For the sake of simplicity, we will assume a linearly polarized wave. Then, only the component of $\vec{E}$ along the direction of polarization, and the corresponding component of $\vec{\mu}$ will be involved. Hence, we suppose that the operator $\vec{\mu}=\mu \vec{e}_{x}$, where $\vec{e}_{x}$ is the unitary vector along the $x$-axis. Also, we have $\vec{E}=E \vec{e}_{x}$, and $\vec{P}=P \vec{e}_{x}$. Note that $\vec{E}$ and $\vec{\mu}$ in Eq. (3) are replaced with scalar quantities $E$ and $\mu$, in which the matrix

$\mu=\left(\mu_{n m}\right)_{(n, m) \in[1, N]}$,

is Hermitian, i.e. $\mu_{m n}=\mu_{n m}^{*}$, where the star denotes the complex conjugate. Due to centrosymmetry, since $\mu_{m n}$ are the matrix elements of an odd operator, the matrix $\mu$ is off-diagonal.

We assume that the characteristic pulse frequency $\omega_{w}$ has the same order of magnitude as the inverse of the pulse duration $1 / t_{w}$, and is very small with respect to any resonance frequency

$\Omega_{n m}=\omega_{n}-\omega_{m}$

in the atomic spectrum, i.e.

$1 / t_{w} \sim \omega_{\omega} \ll \Omega_{n m}$ for all $n, m$.

This motivates the use of the long-wave approximation [65]. We thus introduce the slow variables

$\tau=\varepsilon\left(t-\frac{z}{V}\right)$

$\zeta=\varepsilon^{3} z$

so that we obtain

$\partial_{t}=\varepsilon \partial_{\tau}$,

$\partial_{z}=-\frac{\varepsilon}{V} \partial_{\tau}+\varepsilon^{3} \partial_{\zeta}$

The scaling (Eqs. (9)-(10)) clearly assumes unidirectional propagation. Accurate studies [56,58] proved that this assumption may lead to erroneous results in non-homogeneous media, since some reflections which are negligible for long pulses are not for few-cycle pulses. This must be taken into account in the interpretation of our results, the input wave should indeed be the pulse after it has entered the homogeneous nonlinear medium, which may appreciably differ from the incident pulse.

The field $E$, the polarization density $P$ and the density matrix $\rho$ are expanded in a power series of some small parameter $\varepsilon$ as

$E=\sum_{p \geq 1} \varepsilon^{p} E_{p}$

$P=\sum_{p \geq 1} \varepsilon^{p} P_{p}$ 
$\rho=\sum_{p \geq 0} \varepsilon^{p} \rho^{(p)}$

As $t \rightarrow-\infty, \rho^{(0)}$ is assumed to be the density matrix at thermal equilibrium, while $E_{p}, P_{p}$, and $\rho^{(p)}$ vanish, for any $p \geq 1$.

\subsection{Order 0}

The Schrödinger-von Neumann equation (2) at order $\varepsilon^{0}$ is

$0=\left[H_{0}, \rho^{(0)}\right]$

and

$\left(\left[H_{0}, \rho\right]\right)_{n m}=\hbar \Omega_{n m} \rho_{n m}$.

We assume that each level is non-degenerated, and hence $\Omega_{n m} \neq 0$ if $n \neq m$. Then Eq. (16) yields $\rho_{n m}^{(0)}=0$ for $n \neq m(i, j=1,2, . ., N)$.

\subsection{Order 1}

The Schrödinger-von Neumann equation (2) at order $\varepsilon^{1}$ is

$i \hbar \partial_{\tau} \rho^{(0)}=\left[H_{0}, \rho_{1}\right]-E_{1}\left[\mu, \rho^{(0)}\right]$.

Since

$([\mu, \rho])_{n m}=\sum_{\nu=1}^{N}\left(\mu_{n \nu} \rho_{\nu m}-\rho_{n \nu} \mu_{\nu m}\right)$,

and using Eq. (17), we obtain the equation

$i \hbar \partial_{\tau} \rho_{n m}^{(0)}=\hbar \Omega_{n m} \rho_{n m}^{(1)}-E_{1} \sum_{\nu=1}^{N}\left(\mu_{n \nu} \rho_{\nu m}^{(0)}-\rho_{n \nu}^{(0)} \mu_{\nu m}\right)$,

(recall that $\Omega_{n m}=\omega_{n}-\omega_{m}$ ).

Since $\rho^{(0)}$ is diagonal, Eq. (20) reduces to

$\rho_{n m}^{(1)}=\frac{\mu_{n m} E_{1}\left(\rho_{m m}^{(0)}-\rho_{n n}^{(0)}\right)}{\hbar \Omega_{n m}}$

for $n \neq m$, while the diagonal components $\rho_{n n}^{0}$ are constant.

The polarization density is given by Eq. (5), i.e., at order $\varepsilon^{1}$, by

$P_{1}=N \sum_{n m} \rho_{n m}^{(1)} \mu_{m n}$

Since $\mu$ is off-diagonal, the sum in Eq. (22) extends over $n \neq m$ only. Reporting Eq. (21) into Eq. (22), and using the fact that $\mu$ is Hermitian, we get

$P_{1}=N E_{1} \sum_{n \neq m} \frac{\left|\mu_{n m}\right|^{2}\left(\rho_{m m}^{(0)}-\rho_{n n}^{(0)}\right)}{\hbar \Omega_{n m}}$.

The Maxwell-Helmholtz wave equation (4) at leading order $\varepsilon^{3}$ is

$\frac{1}{V^{2}} \partial_{\tau}^{2} E_{1}=\frac{1}{c^{2}} \partial_{\tau}^{2}\left(E_{1}+\frac{P_{1}}{\varepsilon_{0}}\right)$

Reporting Eq. (23) into Eq. (24) yields

$\frac{1}{V^{2}} \partial_{\tau}^{2} E_{1}=\frac{1}{c^{2}} \partial_{\tau}^{2}\left[E_{1}+\frac{N E_{1}}{\varepsilon_{0} \hbar} \sum_{n \neq m} \frac{\left|\mu_{n m}\right|^{2}\left(\rho_{m m}^{(0)}-\rho_{n n}^{(0)}\right)}{\Omega_{n m}}\right]$.
Eq. (25) admits a nonzero solution if $V=c / n_{0}$, in which the refractive index $n_{0}$ is

$n_{0}=\sqrt{1+\frac{N}{\varepsilon_{0} \hbar} \sum_{n \neq m} \frac{\left|\mu_{n m}\right|^{2}\left(\rho_{m m}^{(0)}-\rho_{n n}^{(0)}\right)}{\Omega_{n m}}}$

From Eq. (26), we can deduce the linear susceptibility $\chi^{(1)}$, which is related to the refractive index through $n_{0}=\sqrt{1+\chi^{(1)}}$, as

$\chi^{(1)}=\frac{N}{\varepsilon_{0} \hbar} \sum_{n \neq m} \frac{\left|\mu_{n m}\right|^{2}\left(\rho_{m m}^{(0)}-\rho_{n n}^{(0)}\right)}{\Omega_{n m}}$.

Eq. (27) is worth being compared to the known expression of the linear susceptibility, which is given in Ref. [66] (Eq. 3.5.15, p. 163):

$\chi_{n m}^{(1)}\left(\omega_{p}\right)=\frac{N}{\varepsilon_{0} \hbar} \sum_{n m}\left(\rho_{m m}^{(0)}-\rho_{n n}^{(0)}\right) \frac{\mu_{m n}^{i} \mu_{n m}^{j}}{\Omega_{n m}-\omega_{p}-i \gamma_{n m}}$.

Here we restrict to a linear polarization, hence $\chi^{(1)}=\chi_{x x}^{(1)}$ and $\mu_{m n}=\mu_{m n}^{x}$. We neglect the damping, i.e. $\gamma_{n m}=0$, and due to the long-wave approximation, the susceptibility must be evaluated as $\omega_{p}$ tends to zero. Then Eq. (27) coincides with Eq. (28).

\subsection{Order 2}

The Schrödinger-von Neumann equation (2) at order $\varepsilon^{2}$ is

$i \hbar \partial_{\tau} \rho^{(1)}=\left[H_{0}, \rho^{(2)}\right]-E_{2}\left[\mu, \rho^{(0)}\right]-E_{1}\left[\mu, \rho^{(1)}\right]$.

Inserting Eq. (17) into Eq. (29), we get the equation

$i \hbar \partial_{\tau} \rho_{n m}^{(1)}=\hbar \Omega_{n m} \rho_{n m}^{(2)}-E_{2} \sum_{\nu=1}^{N}\left(\mu_{n \nu} \rho_{\nu m}^{(0)}-\rho_{n \nu}^{(0)} \mu_{\nu m}\right)-E_{1} \sum_{\nu=1}^{N}\left(\mu_{n \nu} \rho_{\nu m}^{(1)}-\rho_{n \nu}^{(1)} \mu_{\nu m}\right)$.

Since $\rho^{(0)}$ is diagonal, Eq. (30) reduces to

$i \hbar \partial_{\tau} \rho_{n m}^{(1)}=\hbar \Omega_{n m} \rho_{n m}^{(2)}-E_{2} \mu_{n m}\left(\rho_{m m}^{(0)}-\rho_{n n}^{(0)}\right)-E_{1} \sum_{\nu=1}^{N}\left(\mu_{n \nu} \rho_{\nu m}^{(1)}-\rho_{n \nu}^{(1)} \mu_{\nu m}\right)$.

For $n=m$, by using Eq. (21), Eq. (31) becomes

$i \hbar \partial_{\tau} \rho_{n n}^{(1)}=-E_{1}^{2} \sum_{\nu \neq n}\left[\frac{\mu_{n \nu} \mu_{\nu n}\left(\rho_{n n}^{(0)}-\rho_{\nu \nu}^{(0)}\right)}{\hbar \Omega_{\nu n}}-\frac{\mu_{\nu n} \mu_{n \nu}\left(\rho_{\nu \nu}^{(0)}-\rho_{n n}^{(0)}\right)}{\hbar \Omega_{n \nu}}\right]$.

The right-hand-side of Eq. (32) is zero, and hence $\rho_{n n}^{(1)}=0$ for all $n$. Consequently, taking into account the fact that $\mu$ is off-diagonal, we get from Eq. (31)

$$
\begin{aligned}
\rho_{n m}^{(2)}= & \frac{i}{\Omega_{n m}} \partial_{\tau} \rho_{n m}^{(1)}+\frac{E_{2}}{\hbar \Omega_{n m}} \mu_{n m}\left(\rho_{m m}^{(0)}-\rho_{n n}^{(0)}\right) \\
& +\frac{E_{1}}{\hbar \Omega_{n m}} \sum_{\nu \neq n, m}\left(\mu_{n \nu} \rho_{\nu m}^{(1)}-\rho_{n \nu}^{(1)} \mu_{\nu m}\right),
\end{aligned}
$$


for $n \neq m$, i.e., inserting Eq. (21) into Eq. (33) we get,

$$
\begin{aligned}
\rho_{n m}^{(2)}= & \frac{i \mu_{n m}\left(\rho_{m m}^{(0)}-\rho_{n n}^{(0)}\right)}{\hbar \Omega_{n m}^{2}} \partial_{\tau} E_{1}+\frac{E_{2} \mu_{n m}\left(\rho_{m m}^{(0)}-\rho_{n n}^{(0)}\right)}{\hbar \Omega_{n m}} \\
& -\frac{E_{1}^{2}}{\hbar^{2} \Omega_{n m}} \sum_{\nu \neq n, m} \mu_{n \nu} \mu_{\nu m}\left[\frac{\left(\rho_{\nu \nu}^{(0)}-\rho_{n n}^{(0)}\right)}{\Omega_{n \nu}}-\frac{\left(\rho_{m m}^{(0)}-\rho_{\nu \nu}^{(0)}\right)}{\Omega_{\nu m}}\right] .
\end{aligned}
$$

The polarization density at order $\varepsilon^{2}$ is thus

$$
\begin{aligned}
P_{2}= & \frac{i N}{\hbar} \partial_{\tau} E_{1} \sum_{n, m, n \neq m} \frac{\mu_{n m} \mu_{m n}\left(\rho_{m m}^{(0)}-\rho_{n n}^{(0)}\right)}{\Omega_{n m}^{2}} \\
& +\frac{N E_{2}}{\hbar} \sum_{n, m, n \neq m} \frac{\mu_{n m} \mu_{m n}\left(\rho_{m m}^{(0)}-\rho_{n n}^{(0)}\right)}{\Omega_{n m}} \\
& +\frac{N E_{1}^{2}}{\hbar^{2}} \sum_{m, n, \nu}\left[\left(\rho_{m m}^{(0)}-\rho_{\nu v}^{(0)}\right) \frac{\mu_{m n} \mu_{n \nu} \mu_{\nu m}}{\Omega_{n m} \Omega_{\nu m}}\right. \\
& \left.-\left(\rho_{\nu \nu}^{(0)}-\rho_{n n}^{(0)}\right) \frac{\mu_{m n} \mu_{\nu m} \mu_{n v}}{\Omega_{n m} \Omega_{n v}}\right] .
\end{aligned}
$$

The second order nonlinear polarization is thus given by

$P_{2}^{N L}=\varepsilon_{0} \chi^{(2)}\left(E_{1}\right)^{2}$

where the second order susceptibility $\chi^{(2)}$ is given by

$$
\chi^{(2)}=\frac{N}{\varepsilon_{0} \hbar^{2}} \sum_{\substack{m, n, \nu \\ m \neq n \neq v}}\left[\left(\rho_{m m}^{(0)}-\rho_{\nu \nu}^{(0)}\right) \frac{\mu_{m n} \mu_{n \nu} \mu_{\nu m}}{\Omega_{n m} \Omega_{\nu m}}-\left(\rho_{\nu \nu}^{(0)}-\rho_{n n}^{(0)}\right) \frac{\mu_{m n} \mu_{\nu m} \mu_{n \nu}}{\Omega_{n m} \Omega_{n \nu}}\right] .
$$

The expression of $\chi^{(2)}$ can be found in Ref. [66] (Eq. 3.6.14 p. 173), as

$$
\begin{aligned}
& \chi_{i j k}^{(2)}\left(\omega_{p}+\omega_{q} ; \omega_{q}, \omega_{p}\right)=\frac{N}{2 \epsilon_{0} \hbar^{2}} \\
& \times \sum_{m n v}\left\{( \rho _ { m m } ^ { ( 0 ) } - \rho _ { \nu v } ^ { ( 0 ) } ) \left[\frac{\mu_{m n}^{i} \mu_{n \nu}^{j} \mu_{\nu m}^{k}}{\left(\Omega_{n m}-\omega_{p}-\omega_{q}-i \gamma_{n m}\right)\left(\Omega_{\nu m}-\omega_{p}-i \gamma_{\nu m}\right)}\right.\right. \\
& \left.+\frac{\mu_{m n}^{i} \mu_{n \nu}^{k} \mu_{\nu m}^{j}}{\left(\Omega_{n m}-\omega_{p}-\omega_{q}-i \gamma_{n m}\right)\left(\Omega_{\nu m}-\omega_{q}-i \gamma_{\nu m}\right)}\right] \\
& -\left(\rho_{\nu \nu}^{(0)}-\rho_{n n}^{(0)}\right)\left[\frac{\mu_{m n}^{i} \mu_{\nu m}^{j} \mu_{n \nu}^{k}}{\left(\Omega_{n m}-\omega_{p}-\omega_{q}-i \gamma_{n m}\right)\left(\Omega_{n \nu}-\omega_{p}-i \gamma_{n \nu}\right)}\right. \\
& \left.\left.+\frac{\mu_{m n}^{i} \mu_{\nu m}^{k} \mu_{n v}^{j}}{\left(\Omega_{n m}-\omega_{p}-\omega_{q}-i \gamma_{n m}\right)\left(\Omega_{n \nu}-\omega_{q}-i \gamma_{n \nu}\right)}\right]\right\}
\end{aligned}
$$

According to the chosen polarization, we should have

$$
\chi^{(2)}=\chi_{x x x}^{(2)}(2 \omega ; \omega, \omega)
$$

evaluated as $\omega$ tends to zero, and neglecting the damping $\left(\gamma_{n m}=0\right)$. It is seen that Eqs. (36) and (38) coincide under these conditions, taking into account the fact that $\mu$ is off-diagonal. In the present study, we assume a centrosymmetric medium, and hence $\chi^{(2)}=0$. The coefficient of $\partial_{\tau} E_{1}$ in Eq. (35) is

$A=i N \sum_{n m} \frac{\mu_{n m} \mu_{m n}\left(\rho_{m m}^{(0)}-\rho_{n n}^{(0)}\right)}{\hbar \Omega_{n m}^{2}}$

Permuting the dummy subscripts $m$ and $n$ changes the sign of the term in the latter sum, hence $A=0$.

The Maxwell-Helmholtz wave equation (4) at order $\varepsilon^{4}$ is

$\frac{1}{V^{2}} \partial_{\tau}^{2} E_{2}=\frac{1}{c^{2}} \partial_{\tau}^{2}\left(E_{2}+\frac{P_{2}}{\varepsilon_{0}}\right)$

which is automatically satisfied if the expression (Eq. (26)) of the refractive index is taken into account.

\subsection{Order 3}

The Schrödinger-von Neumann equation (2) at order $\varepsilon^{3}$ is

$i \hbar \partial_{\tau} \rho^{(2)}=\left[H_{0}, \rho^{(3)}\right]-E_{3}\left[\mu, \rho^{(0)}\right]-E_{2}\left[\mu, \rho^{(1)}\right]-E_{1}\left[\mu, \rho^{(2)}\right]$.

Using Eqs. (17) and (19) into Eq. (42), we obtain the equation

$$
\begin{aligned}
i \hbar \partial_{\tau} \rho_{n m}^{(2)}= & \hbar \Omega_{n m} \rho_{n m}^{(3)}-E_{3} \sum_{\nu=1}^{N}\left(\mu_{n \nu} \rho_{\nu m}^{(0)}-\rho_{n \nu}^{(0)} \mu_{\nu m}\right) \\
& -E_{2} \sum_{\nu=1}^{N}\left(\mu_{n \nu} \rho_{\nu m}^{(1)}-\rho_{n \nu}^{(1)} \mu_{\nu m}\right)-E_{1} \sum_{\nu=1}^{N}\left(\mu_{n \nu} \rho_{\nu m}^{(2)}-\rho_{n \nu}^{(2)} \mu_{\nu m}\right) .
\end{aligned}
$$

A first step is to compute the diagonal terms $\rho_{n n}^{(2)}$. For $m=n$, Eq. (43) becomes

$i \hbar \partial_{\tau} \rho_{n n}^{(2)}=-S_{1 n} E_{2}-S_{2 n} E_{1}$,

where we have set

$S_{j n}=\sum_{\nu \neq n}\left(\mu_{n \nu} \rho_{\nu n}^{(j)}-\rho_{n \nu}^{(j)} \mu_{\nu n}\right)$

for $j=1,2 . S_{1 n}$ contains the same expression as the right-hand-side term of Eq. (32) above, and consequently $S_{1 n}=0$. Using the expression (34) of $\rho_{n \nu}^{(2)}$ for $n \neq \nu, S_{2 n}$ can be expanded as

$S_{2 n}=F_{1 n} E_{2}+F_{2 n} \partial_{\tau} E_{1}+F_{3 n} E_{1}^{2}$

where

$F_{1 n}=\frac{\mu_{\nu n} \mu_{n \nu}}{\hbar}\left[\frac{\left(\rho_{n n}^{(0)}-\rho_{\nu v}^{(0)}\right)}{\Omega_{\nu n}}+\frac{\left(\rho_{\nu \nu}^{(0)}-\rho_{n n}^{(0)}\right)}{\Omega_{\nu n}}\right]=0$

as $S_{1 n}=0$ above, and

$F_{2 n}=\sum_{\nu \neq n} \frac{2 i \mu_{n \nu} \mu_{v n}}{\hbar \Omega_{v n}^{2}}\left(\rho_{n n}^{(0)}-\rho_{v v}^{(0)}\right)$ 


$$
\begin{aligned}
F_{3 n} & =\sum_{\nu \neq n}^{N}\left\{\frac{\mu_{n \nu}}{\hbar \Omega_{\nu n}} \sum_{l \neq n, \nu}^{N} \mu_{l n} \mu_{\nu l}\left[\frac{\left(\rho_{n n}^{(0)}-\rho_{l l}^{(0)}\right)}{\hbar \Omega_{l n}}-\frac{\left(\rho_{l l}^{(0)}-\rho_{\nu v}^{(0)}\right)}{\hbar \Omega_{\nu l}}\right]\right. \\
& \left.-\frac{\mu_{\nu n}}{\hbar \Omega_{n \nu}} \sum_{l=1}^{N} \mu_{l \nu} \mu_{n l}\left[\frac{\left(\rho_{v \nu}^{(0)}-\rho_{l l}^{(0)}\right)}{\hbar \Omega_{l v}}-\frac{\left(\rho_{l l}^{(0)}-\rho_{n n}^{(0)}\right)}{\hbar \Omega_{n l}}\right]\right\} .
\end{aligned}
$$

Then we get

$\rho_{n n}^{(2)}=\frac{i F_{2 n}}{2 \hbar} E_{1}^{2}+\frac{i F_{3 n}}{\hbar} \int_{-\infty}^{\tau} E_{1}^{3} d \tau$

The off-diagonal terms of $\rho^{(3)}$ can also be computed. Since $\rho^{(0)}$ is a diagonal matrix, and $\mu$ and $\rho^{(1)}$ are off-diagonal matrices, Eq. (43) yields, for $n \neq m$,

$$
\begin{aligned}
\rho_{n m}^{(3)}= & \frac{i}{\Omega_{n m}} \partial_{\tau} \rho_{n m}^{(2)}+\frac{E_{3} \mu_{n m}}{\hbar \Omega_{n m}}\left(\rho_{m m}^{(0)}-\rho_{n n}^{(0)}\right)+\frac{E_{2}}{\hbar \Omega_{n m}} \sum_{\nu \neq m, n}\left(\mu_{n \nu} \rho_{\nu m}^{(1)}-\rho_{n \nu}^{(1)} \mu_{\nu m}\right) \\
& +\frac{E_{1}}{\hbar \Omega_{n m}} \sum_{\nu \neq m, n}\left(\mu_{n \nu} \rho_{\nu m}^{(2)}-\rho_{n \nu}^{(2)} \mu_{\nu m}\right)+\frac{E_{1} \mu_{n m}}{\hbar \Omega_{n m}}\left(\rho_{m m}^{(2)}-\rho_{n n}^{(2)}\right) .
\end{aligned}
$$

Reporting Eqs. (21), (34) and (51) into Eq. (52), we compute the polarization density as

$$
\begin{aligned}
P_{3}= & \varepsilon_{0} \chi^{(1)} E_{3}+2 \varepsilon_{0} \chi^{(2)} E_{1} E_{2}+\varepsilon_{0} \chi^{(3)}\left(E_{1}\right)^{3} \\
& +A \partial_{\tau} E_{2}+B \partial_{\tau}^{2} E_{1}+C E_{1} \partial_{\tau} E_{1}+D E_{1} \int_{-\infty}^{\tau} E_{1}^{3} d \tau,
\end{aligned}
$$

in which $\chi^{(1)}$ is given by Eq. (27), and $\chi^{(2)}$ is given by Eq. (38). Here $\chi^{(3)}=\chi_{R}^{(3)}+\chi_{S}^{(3)}$, with

$$
\begin{aligned}
\chi_{R}^{(3)}= & \frac{N}{\varepsilon_{0} \hbar^{3}} \sum_{n m \nu l}\left[\frac{\mu_{m n} \mu_{n \nu} \mu_{l m} \mu_{\nu l}\left(\rho_{m m}^{(0)}-\rho_{l l}^{(0)}\right)}{\Omega_{n m} \Omega_{\nu m} \Omega_{l m}}-\frac{\mu_{m n} \mu_{n \nu} \mu_{l m} \mu_{\nu l}\left(\rho_{l l}^{(0)}-\rho_{\nu \nu}^{(0)}\right)}{\Omega_{n m} \Omega_{\nu m} \Omega_{\nu l}}\right. \\
& \left.-\frac{\mu_{m n} \mu_{\nu m} \mu_{l \nu} \mu_{n l}\left(\rho_{\nu \nu}^{(0)}-\rho_{l l}^{(0)}\right)}{\Omega_{n m} \Omega_{n \nu} \Omega_{l \nu}}+\frac{\mu_{m n} \mu_{\nu m} \mu_{l \nu} \mu_{n l}\left(\rho_{l l}^{(0)}-\rho_{n n}^{(0)}\right)}{\Omega_{n m} \Omega_{n \nu} \Omega_{n l}}\right],
\end{aligned}
$$

the sum being extended on all terms for which the denominators do not vanish (the 'regular' terms, motivating the subscript ' $R$ '), and

$\chi_{S}^{(3)}=\frac{N}{\varepsilon_{0} \hbar} \sum_{n, m, n \neq m} \frac{\mu_{m n} \mu_{n m}}{\Omega_{n m}}\left(\frac{i F_{2 m}}{2 \hbar}-\frac{i F_{2 n}}{2 \hbar}\right)$,

where $F_{2 n}$ is given by Eq. (48). The coefficient $A$ is given by Eq. (40),

$$
\begin{aligned}
B= & \frac{-N}{\hbar} \sum_{n m} \frac{\mu_{n m} \mu_{m n}\left(\rho_{m m}^{(0)}-\rho_{n n}^{(0)}\right)}{\Omega_{n m}^{3}}, \\
C= & -\frac{i N}{\hbar^{2}} \sum_{n m \nu}\left[\frac{\mu_{m n} \mu_{n \nu} \mu_{\nu m}\left(\rho_{m m}^{(0)}-\rho_{\nu v}^{(0)}\right)}{\Omega_{n m} \Omega_{\nu m}^{2}}-\frac{\mu_{m n} \mu_{n \nu} \mu_{\nu m}\left(\rho_{\nu \nu}^{(0)}-\rho_{n n}^{(0)}\right)}{\Omega_{n m} \Omega_{n \nu}^{2}}\right] \\
& +\frac{2 i N}{\hbar^{2}} \sum_{n m \nu}\left[\frac{\mu_{m n} \mu_{\nu m} \mu_{n \nu}\left(\rho_{\nu \nu}^{(0)}-\rho_{n n}^{(0)}\right)}{\Omega_{n m}^{2} \Omega_{n \nu}}-\frac{\mu_{m n} \mu_{\nu m} \mu_{n \nu}\left(\rho_{m m}^{(0)}-\rho_{\nu \nu}^{(0)}\right)}{\Omega_{n m}^{2} \Omega_{\nu m}}\right],
\end{aligned}
$$

and

$D=\frac{N}{\hbar} \sum_{n, m, n \neq m} \frac{\mu_{m n} \mu_{n m}}{\Omega_{n m}}\left(\frac{i F_{3 m}}{2 \hbar}-\frac{i F_{3 n}}{2 \hbar}\right)$,

where $F_{3 n}$ is given by Eq. (49).
Due to the centrosymmetry, the coefficient $C$ is zero. Consider indeed the second order susceptibility $\chi_{x x x}^{(2)}\left(\omega_{p}+\omega_{q} ; \omega_{q}, \omega_{p}\right)$ given by Eq. (38), for $\omega_{p}=\omega_{q}=\omega$, i.e.

$$
\begin{aligned}
\chi^{(2)}(2 \omega ; \omega, \omega)= & \frac{N}{\epsilon_{0} \hbar^{2}} \sum_{m n \nu}\left[\left(\rho_{m m}^{(0)}-\rho_{\nu v}^{(0)}\right) \frac{\mu_{m n} \mu_{n \nu} \mu_{\nu m}}{\left(\Omega_{n m}-2 \omega\right)\left(\Omega_{\nu m}-\omega\right)}\right. \\
& \left.-\left(\rho_{\nu \nu}^{(0)}-\rho_{n n}^{(0)}\right) \frac{\mu_{m n} \mu_{\nu m} \mu_{n \nu}}{\left(\Omega_{n m}-2 \omega\right)\left(\Omega_{n \nu}-\omega\right)}\right] .
\end{aligned}
$$

Taking the derivative with respect to $\omega$, and then the limit as $\omega$ tends to zero yields

$$
\begin{aligned}
\left.\frac{d}{d \omega} \chi^{(2)}(2 \omega ; \omega, \omega)\right|_{\omega=0}= & \frac{N}{\epsilon_{0} \hbar^{2}} \sum_{m n \nu}\left[\left(\rho_{m m}^{(0)}-\rho_{\nu \nu}^{(0)}\right) \frac{\mu_{m n} \mu_{n \nu} \mu_{\nu m}}{\Omega_{n m} \Omega_{\nu m}^{2}}\right. \\
& \left.-\left(\rho_{\nu \nu}^{(0)}-\rho_{n n}^{(0)}\right) \frac{\mu_{m n} \mu_{\nu m} \mu_{n \nu}}{\Omega_{n m} \Omega_{n \nu}^{2}}\right] \\
& +\frac{2 N}{\epsilon_{0} \hbar^{2}} \sum_{m n \nu}\left[\left(\rho_{m m}^{(0)}-\rho_{\nu \nu}^{(0)}\right) \frac{\mu_{m n} \mu_{n \nu} \mu_{\nu m}}{\Omega_{n m}^{2} \Omega_{\nu m}}\right. \\
& \left.-\left(\rho_{\nu \nu}^{(0)}-\rho_{n n}^{(0)}\right) \frac{\mu_{m n} \mu_{\nu m} \mu_{n \nu}}{\Omega_{n m}^{2} \Omega_{n \nu}}\right] .
\end{aligned}
$$

It is seen that

$\left.\frac{d}{d \omega} \chi^{(2)}(2 \omega ; \omega, \omega)\right|_{\omega=0}=\frac{-i C}{\varepsilon_{0}}$.

The medium being centrosymmetric, $\chi^{(2)}(2 \omega ; \omega, \omega) \equiv 0$, and hence $C=0$. It has also been seen that $A=0$ and $\chi^{(2)}=0$. The coefficient $D$ is a nonlinear term of the 4 th order. Since the order is even, it must be zero due to centrosymmetry. Notice that this feature cannot be justified for the expression of the coefficient itself, as it was the case for the $\chi^{(2)}$ coefficient. Hence $D=0$ and finally the polarization density reduces to

$P_{3}=\varepsilon_{0} \chi^{(1)} E_{3}+\varepsilon_{0} \chi^{(3)}\left(E_{1}\right)^{3}+B \partial_{\tau}^{2} E_{1}$. $\varepsilon^{5}$. It is

Consider now the Maxwell-Helmholtz wave equation (4) at order

$\frac{1}{V^{2}} \partial_{\tau}^{2} E_{3}-\frac{2}{V} \partial_{\zeta} \partial_{\tau} E_{1}=\frac{1}{c^{2}} \partial_{\tau}^{2}\left(E_{3}+\frac{P_{3}}{\varepsilon_{0}}\right)$

Reporting Eq. (51), and using the expression (26) of the velocity $V$, the terms involving $E_{3}$ vanish, and Eq. (62) reduces to

$\partial_{\zeta} E_{1}+\gamma \partial_{\tau}\left(E_{1}\right)^{3}+\beta \partial_{\tau}^{3} E_{1}=0$

which is exactly the mKdV equation.

The nonlinear coefficient is

$\gamma=\frac{1}{2 n_{0} c} \chi^{(3)}$

with $\chi^{(3)}$ given by Eqs. (53)-(54). An expression of $\chi^{(3)}$ can be found in Ref. [66] (Eq. (3.7.10), p. 182), as

$\chi_{\text {kjih }}^{(3)}\left(\omega_{p}+\omega_{q}+\omega_{r} ; \omega_{r}, \omega_{q}, \omega_{p}\right)=\mathcal{P}_{I}\left(\tilde{\chi}_{k j i h}^{(3)}\left(\omega_{p}+\omega_{q}+\omega_{r} ; \omega_{r}, \omega_{q}, \omega_{p}\right)\right)$, 
in which $\mathcal{P}_{I}$ represents an averaging over all permutations of $\omega_{r}, \omega_{q}$ and $\omega_{p}$, and

$$
\begin{aligned}
& \tilde{\chi}_{k j i h}^{(3)}\left(\omega_{p}+\omega_{q}+\omega_{r} ; \omega_{r}, \omega_{q}, \omega_{p}\right)=\frac{N}{\varepsilon \hbar^{3}} \sum_{n m \nu l} \\
& {\left[\frac{\left(\rho_{m m}^{(0)}-\rho_{l l}^{(0)}\right) \mu_{m n}^{k} \mu_{n \nu}^{j} \mu_{\nu l}^{i} \mu_{l m}^{h}}{\left(\Omega_{n m}-\omega_{p}-\omega_{q}-\omega_{r}\right)\left(\Omega_{\nu m}-\omega_{p}-\omega_{q}\right)\left(\Omega_{l m}-\omega_{p}\right)}\right.} \\
& -\frac{\left(\rho_{l l}^{(0)}-\rho_{\nu \nu}^{(0)}\right) \mu_{m n}^{k} \mu_{n \nu}^{j} \mu_{l m}^{i} \mu_{\nu l}^{h}}{\left(\Omega_{n m}-\omega_{p}-\omega_{q}-\omega_{r}\right)\left(\Omega_{\nu m}-\omega_{p}-\omega_{q}\right)\left(\Omega_{\nu l}-\omega_{p}\right)} \\
& -\frac{\left(\rho_{\nu \nu}^{(0)}-\rho_{l l}^{(0)}\right) \mu_{m n}^{k} \mu_{\nu m}^{j} \mu_{n l}^{i} \mu_{l v}^{h}}{\left(\Omega_{n m}-\omega_{p}-\omega_{q}-\omega_{r}\right)\left(\Omega_{n \nu}-\omega_{p}-\omega_{q}\right)\left(\Omega_{l \nu}-\omega_{p}\right)} \\
& \left.+\frac{\left(\rho_{l l}^{(0)}-\rho_{n n}^{(0)}\right) \mu_{m n}^{k} \mu_{\nu m}^{j} \mu_{l \nu}^{i} \mu_{n l}^{h}}{\left(\Omega_{n m}-\omega_{p}-\omega_{q}-\omega_{r}\right)\left(\Omega_{n \nu}-\omega_{p}-\omega_{q}\right)\left(\Omega_{n l}-\omega_{p}\right)}\right]
\end{aligned}
$$

in which we set the relaxation rates $\gamma_{n m}$ to zero.

The present $\chi^{(3)}$ should coincide with $\chi_{x x x x}^{(3)}(0 ; 0,0,0)$, however several terms in the sum (66) are singular as $\left(\omega_{r}, \omega_{q}, \omega_{p}\right) \rightarrow(0,0,0)$. It is straightforwardly seen that the sum of the regular terms exactly coincides with $\chi_{R}^{(3)}$ as given by Eq. (53). Taking into account the fact that $\mu$ is off-diagonal, the singular terms in the sum (66) are:

- The 1 st term for $\nu=m$ :

$$
A_{m n l}=\frac{-K_{m n l}}{\left(\Omega_{n m}-\omega_{p}-\omega_{q}-\omega_{r}\right)\left(\omega_{p}+\omega_{q}\right)\left(\Omega_{l m}-\omega_{p}\right)},
$$

with

$K_{m n l}=\left(\rho_{m m}^{(0)}-\rho_{l l}^{(0)}\right) \mu_{m n} \mu_{n m} \mu_{m l} \mu_{l m}$.

- The 2nd term for $\nu=m$ :

$B_{m n l}=\frac{K_{m n l}}{\left(\Omega_{n m}-\omega_{p}-\omega_{q}-\omega_{r}\right)\left(\omega_{p}+\omega_{q}\right)\left(\Omega_{l m}+\omega_{p}\right)}$.

- The 3rd term for $\nu=n$ :

$C_{m n l}=\frac{K_{n m l}}{\left(\Omega_{n m}-\omega_{p}-\omega_{q}-\omega_{r}\right)\left(\omega_{p}+\omega_{q}\right)\left(\Omega_{l n}-\omega_{p}\right)}$.

- The 4 th term for $\nu=n$ :

$D_{m n l}=\frac{-K_{n m l}}{\left(\Omega_{n m}-\omega_{p}-\omega_{q}-\omega_{r}\right)\left(\omega_{p}+\omega_{q}\right)\left(\Omega_{l n}+\omega_{p}\right)}$.

Let us set $\left(\omega_{r}, \omega_{q}, \omega_{p}\right)=\left(-\omega+\delta \omega_{r}, \omega+\delta \omega_{q}, \omega+\delta \omega_{p}\right)$, in which $\delta \omega_{r}$, $\delta \omega_{q}$ and $\delta \omega_{p}$ are infinitesimal quantities. After some calculations, the infinitesimal quantities simplify and we get

$\mathcal{P}_{I}\left(A_{m n l}+B_{m n l}\right)=\frac{-K_{m n l}}{3\left(\Omega_{n m}-\omega\right)}\left[\frac{1}{\left(\Omega_{l m}+\omega\right)^{2}}+\frac{1}{\left(\Omega_{l m}-\omega\right)^{2}}+\frac{1}{\Omega_{l m}^{2}-\omega^{2}}\right]$.
It is comparable to the expression of $\chi^{(3)}$ found in Ref. [67], but with a slight sign change. As $\omega \rightarrow 0$, it yields

$\mathcal{P}_{I}\left(A_{m n l}+B_{m n l}\right)=\frac{-\left(\rho_{m m}^{(0)}-\rho_{l l}^{(0)}\right) \mu_{m n} \mu_{n m} \mu_{m l} \mu_{l m}}{\Omega_{n m} \Omega_{l m}^{2}}$.

$\mathcal{P}_{I}\left(C_{m n l}+D_{m n l}\right)$ yields the same expression as Eq. (73) with permuted $n$ and $m$. On the other hand, Eqs. (54) and (48) yield

$\chi_{S}^{(3)}=\frac{-2 N}{\varepsilon_{0} \hbar^{3}} \sum_{\substack{n, m, \nu, n \neq m, \nu \neq m}} \frac{\mu_{m n} \mu_{n m} \mu_{\nu m} \mu_{m \nu}}{\Omega_{n m} \Omega_{\nu m}^{2}}\left(\rho_{m m}^{(0)}-\rho_{\nu \nu}^{(0)}\right)$

and hence

$\chi_{S}^{(3)}=\frac{N}{\varepsilon_{0} \hbar^{3}} \sum_{\substack{n, m, \nu, n \neq m, \nu \neq m}} \mathcal{P}_{I}\left(A_{m n l}+B_{m n l}+C_{m n l}+D_{m n l}\right)$.

As a conclusion, taking into account both regular and singular terms, we see that $\chi^{(3)}$ exactly coincides with $\chi_{x x x x}^{(3)}(0 ; 0,0,0)$.

The expression (Eq. (64)) of the nonlinear coefficient slightly differs from the analogous expression found in Refs. [34,39]. A factor $4 \pi$ is due to the system of units (i.e., CGS units versus SI units). In fact, the value of $\chi^{(3)}$ given by Eq. (78) in Ref. [67] is smaller by a factor of $1 / 3$. The expression of the nonlinear coefficient in [34] was based on this value of $\chi^{(3)}$, and this initial error resulted in the erroneous coefficient of 6 in Eq. (25) in [34] instead of 2 in Eq. (64) above.

The dispersion coefficient is

$\beta=\frac{1}{2 \varepsilon_{0} n_{0} c} B$

where $B$ is given by Eq. (55). Taking twice the derivative of Eq. (28) with respect to $\omega_{p}$, then setting $\omega_{p}=0$, and comparing the result with Eq. (55) shows that

$B=\left.\frac{-\varepsilon_{0}}{2} \frac{d^{2} \chi_{x x}^{(1)}(\omega)}{d \omega^{2}}\right|_{\omega=0}$.

The wave vector is $k(\omega)=n_{0}(\omega) \omega / c$, and its third derivative for $\omega=0$ is

$\left.\frac{d^{3} k}{d \omega^{3}}\right|_{\omega=0}=\frac{3}{c} n_{0}^{\prime \prime}$

where we have set

$n_{0}^{\prime \prime}=\left.\frac{d^{2} n_{0}}{d \omega^{2}}\right|_{\omega=0}$

Taking twice the derivative of $n_{0}=\sqrt{1+\chi^{(1)}}$ with respect to $\omega$ yields

$\frac{d^{2} n_{0}}{d \omega^{2}}=\frac{1}{2 n_{0}} \frac{d^{2} \chi^{(1)}}{d \omega^{2}}-\frac{1}{2 n_{0}^{2}}\left(\frac{d \chi^{(1)}}{d \omega}\right)^{2}$

however, $d \chi^{(1)} / d \omega$ for $\omega=0$ is proportional to the coefficient $A$ given by Eq. (40), hence is zero. Finally, it is found that the dispersion coefficient $\beta$ can be written as

$\beta=\left.\frac{-1}{6} \frac{d^{3} k}{d \omega^{3}}\right|_{\omega=0}$ 
which exactly coincides with the expression found in Ref. [34] and generalizes the latter. The equivalent expression

$\beta=\frac{-n_{0}^{\prime \prime}}{2 c}$

evidences the fact that $\beta$ is not a third-order dispersion as it could been believed at first glance, but accounts in the present approximation for the group velocity dispersion. It also may account for higher order dispersion terms, see Ref. [68].

\section{The breather solution of the modified-Korteweg-de Vries equation}

The mKdV equation (63) is completely integrable by means of the inverse scattering transform [69]. The $N$-soliton solution has been given by Hirota [70]. It is more convenient to write the mKdV equation (63) into the dimensionless form

$\partial_{Z} u+2 \partial_{T} u^{3}+\sigma \partial_{T}^{3} u=0$,

where $\sigma= \pm 1, u$ is a dimensionless electric field, and $Z$ and $T$ dimensionless space and time variables defined relative to the laboratory variables as

$u=\frac{E}{E_{0}} \quad, \quad Z=\frac{z}{L} \quad, \quad T=\frac{t-z / V}{t_{w}}$.

The reference time is thus chosen to be the pulse length $t_{w}$ (in physical units). Recall that the atomic resonance frequencies $\Omega_{n m}$ have been chosen above as zero order quantities in the perturbative scheme, while $t_{w}$ is assumed to be formally large, of order $1 / \varepsilon$, with respect to the zero order times $1 / \Omega_{n m}$. The characteristic electric field and propagation distance are

$E_{0}=\frac{1}{t_{w}} \sqrt{\frac{-2 \sigma n_{0} n_{0}^{\prime \prime}}{\chi^{(3)}}}$,

$L=\frac{2 c t_{w}^{3}}{\left(-\sigma n_{0}^{\prime \prime}\right)}$.

If $\chi^{(3)}$ and $n_{0}^{\prime \prime}$ have opposite sign, which is typically for $\chi^{(3)}>0$ and anomalous dispersion, then $\sigma=+1$, and the mKdV equation (63) is a focusing one. Else, typically for $\chi^{(3)}>0$ and normal dispersion, $\sigma=-1$,

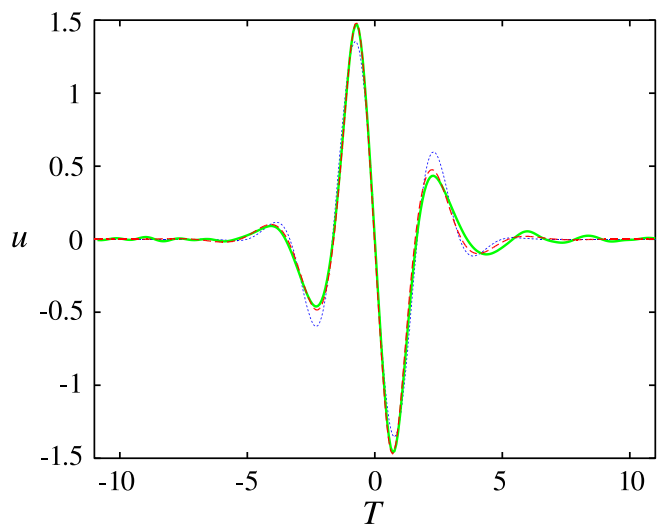

Fig. 1. Propagation of a FCP according to the mKdV equation. Blue dotted line: initial input with Gaussian envelope. Green thick solid line: the FCP soliton observed after some propagation distance $(Z=79.72)$. Dashed red line: fit of the latter by the analytic breather.

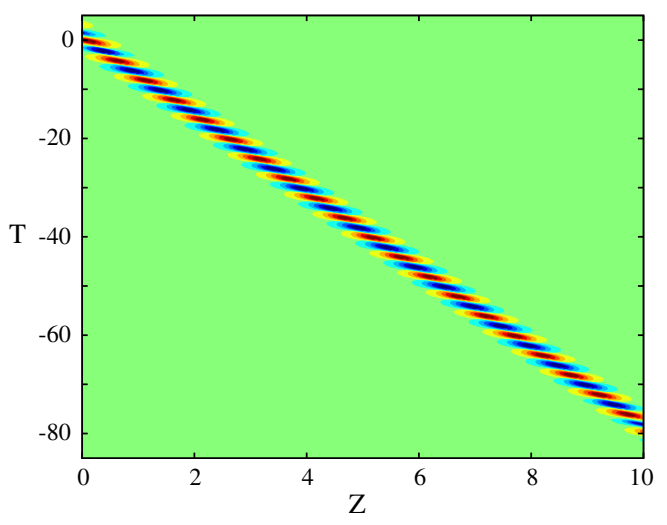

Fig. 2. Propagation of a FCP according to the mKdV equation: $u$ against $T$ and $Z$ computed with the analytic formula (Eq. (88)); parameters are the same as the fit in Fig. 1.

Eq. (63) is a defocusing one and describes nonlinear dispersion [39]. In the focusing case, the mKdV equation admits real single-soliton solutions, and $\mathrm{N}$-soliton and breather solutions. Integrating the $\mathrm{mKdV}$ equation (83) with respect to $T$, under the assumption that $u$, i.e. the electric field, and its derivatives, vanishes at infinity, it is seen that the conservation law

$\partial_{Z} \int_{-\infty}^{+\infty} u d T=0$

is satisfied. This is the expression in our situation of the general law of the conservation of the electric pulse area, as derived in [56,58]. Due to the Galilean transformation and the scaling (9-10), it is seen from Maxwell equations that the magnetic field is $\vec{B}=u E_{0} / V \vec{e}_{y}$, and that $\int_{-\infty}^{+\infty} B_{\alpha} d z \propto \int_{-\infty}^{+\infty} u d T$, hence the conservation law of the magnetic pulse area is also satisfied by the mKdV Eq. (63), since it does not differ from (87).

The two-soliton solution of the mKdV equation is [70]

$u=\frac{e^{\eta_{1}}+e^{\eta_{2}}+\left(\frac{p_{1}-p_{2}}{p_{1}+p_{2}}\right)^{2}\left(\frac{e^{\eta_{1}}}{4 p_{1}^{2}}+\frac{e^{\eta_{2}}}{4 p_{2}^{2}}\right) e^{\eta_{1}+\eta_{2}}}{1+\frac{e^{2 \eta_{1}}}{4 p_{1}^{2}}+\frac{2}{\left(p_{1}+p_{2}\right)^{2}} e^{\eta_{1}+\eta_{2}}+\frac{e^{2 \eta_{2}}}{4 p_{2}^{2}}+\left(\frac{p_{1}-p_{2}}{p_{1}+p_{2}}\right)^{4} \frac{e^{2 \eta_{1}+2 \eta_{2}}}{16 p_{1}^{2} p_{2}^{2}}}$,

with

$\eta_{j}=p_{j} T-p_{j}^{3} Z-\gamma_{j}$

for $j=1$ and 2 . The parameters $p_{1}, p_{2}, \gamma_{1}$, and $\gamma_{2}$ are arbitrary. If $p_{2}=p_{1}^{*}$, where $*$ denotes the complex conjugate, and $\gamma_{2}=\gamma_{1}^{*}$, the explicit solution (88) is an oscillating localized solution, called breather soliton, which actually adequately describes a FCP soliton.

An example of FCP soliton propagation is shown on Fig. 1. The mKdV equation (63) is solved using the exponential time differencing 4th order Runge-Kutta scheme [71], for an input data (blue dotted line) of the form

$u=\mathcal{A} e^{-T^{2} / w^{2}} \sin \left(\omega_{0} T+\phi\right)$,

with the parameters $w=2.5, \mathcal{A}=1.5, \omega_{0}=0.6 \pi$, and $\phi=\pi$. The computation was run until $Z \simeq 80$. The pulse evolves with very few changes in shape and width, apart from periodic oscillations. We chose a propagation distance $(Z=79.72)$ at which the carrier-envelope phase of the final FCP is the same as the initial one, moved to the initial position and plotted it in Fig. 1 (green thick solid line) for comparison. A fit with the breather (88) is also shown (dashed red line): it is very close to the numerical result. The values of the parameters which yield the best fit are $p_{1}=0.875+1.7 i, \gamma_{1}=0.24 i$ (and a small shift in position). 
The propagation of the breather, computed from the analytic formula (Eq. (88)), is shown in Fig. 2. Note that the same picture can be obtained numerically.

\section{Conclusions}

In conclusion, in this paper we have studied in detail the propagation of ultrashort pulses containing only few optical cycles, in a centrosymmetric nonlinear cubic (Kerr) material described by a general Hamiltonian of multilevel atoms. Assuming that all transition frequencies of the material are well above the typical wave frequency, we can use a long-wave approximation to derive an approximate model for nonlinear ultrashort optical pulse propagation in such a medium. The obtained equation is of modified Korteweg-de Vries type and allows the adequate description of FCP soliton propagation in a cubic nonlinear optical medium. However, in a real nonlinear optical medium, a broad transparency range is required, and hence all atomic transitions must be far enough from the pulse central frequency. However in such materials not all transitions belong to the ultraviolet domain. The contribution of the infrared transitions, which are not taken into account in the present work, should be considered in a further study by means of a short-wave approximation applied to the same general quantum model. Therefore a fully realistic model for FCP propagation will be obtained by putting together the results yielded by both long- and short-wave approximations.

\section{Acknowledgments}

The work of DM was supported in part by a Senior Chair Grant from the Région Pays de Loire, France. Support from the Romanian Ministry of Education and Research (Project PN-II-ID-PCE-2011-30083) is also acknowledged.

\section{References}

[1] L. Gallmann, D.H. Sutter, N. Matuschek, G. Steinmeyer, U. Keller, C. Iaconis, I.A. Walmsley, Optics Letters 24 (1999) 1314.

[2] U. Morgner, F.X. Kärtner, S.H. Cho, Y. Chen, H.A. Haus, J.G. Fujimoto, E.P. Ippen, V. Scheuer, G. Angelow, T. Tschudi, Optics Letters 24 (1999) 411.

[3] D.H. Sutter, G. Steinmeyer, L. Gallmann, N. Matuschek, F. Morier-Genoud, U. Keller, V. Scheuer, G. Angelow, T. Tschudi, Optics Letters 24 (1999) 631.

[4] A. Shirakawa, I. Sakane, M. Takasaka, T. Kobayashi, Applied Physics Letters 74 (1999) 2268.

[5] T. Brabek, F. Krausz, Reviews of Modern Physics 72 (2000) 545.

[6] E. Goulielmakis, M. Schultze, M. Hofstetter, V.S. Yakovlev, J. Gagnon, M. Uiberacker, A.L. Aquila, E.M. Gullikson, D.T. Attwood, R. Kienberger, F. Krausz, U. Kleineberg, Science 320 (2008) 1614.

[7] M. Wegener, Extreme Nonlinear Optics, Springer, Berlin, 2005.

[8] A. Scrinzi, M.Yu. Ivanov, R. Kienberger, D.M. Villeneuve, Journal of Physics B 39 (2006) R1.

[9] F. Krausz, M. Ivanov, Reviews of Modern Physics 81 (2009) 163.

[10] X. Tan, X. Fan, Y. Yang, D. Tong, Journal of Modern Optics 55 (2008) 2439.

[11] N.N. Rosanov, V.E. Semenov, N.V. Vyssotina, Laser Physics 17 (2007) 1311.

[12] N.N. Rosanov, V.E. Semenov, N.V. Vysotina, Quantum Electronics 38 (2008) 137.

[13] A. Nazarkin, Physical Review Letters 97 (2006) 163904.

[14] A.I. Maimistov, Quantum Electronics 40 (2010) 756.

[15] T. Brabec, F. Krausz, Physical Review Letters 78 (1997) 3282.

[16] M.V. Tognetti, H.M. Crespo, Journal of the Optical Society of America B 24 (2007) 1410.

[17] A.A. Voronin, A.M. Zheltikov, Physical Review A 78 (2008) 063834.

[18] A. Kumar, V. Mishra, Physical Review A 79 (2009) 063807.

[19] P. Kinsler, G.H.C. New, Physical Review A 67 (2003) 023813.
[20] A.A. Zozulya, S.A. Diddams, T.S. Clement, Physical Review A 58 (1998) 3303

[21] A.A. Zozulya, S.A. Diddams, A.G. Van Engen, T.S. Clement, Physical Review Letters 82 (1999) 1430.

[22] N. Aközbek, M. Scalora, C.M. Bowden, S.L. Chin, Optics Communications 191 (2001) 353.

[23] J.E. Rothenberg, Optics Letters 17 (1992) 1340

[24] J.K. Ranka, A.L. Gaeta, Optics Letters 23 (1998) 534

[25] E.M. Belenov, A.V. Nazarkin, JETP Letters 51 (1990) 288.

[26] A.I. Maimistov, S.O. Elytin, Journal of Modern Optics 39 (1992) 2201.

[27] A.E. Kaplan, P.L. Shkolnikov, Physical Review Letters 75 (1995) 2316.

[28] M.A. Porras, Physical Review A 60 (1999) 5069.

[29] S.V. Sazonov, JETP 92 (2001) 361.

[30] A.I. Maimistov, Optics and Spectroscopy 76 (1994) 569.

[31] I.V. Mel'nikov, D. Mihalache, F. Moldoveanu, N.C. Panoiu, Physical Review A 56 (1997) 1569.

[32] I.V. Mel'nikov, D. Mihalache, F. Moldoveanu, N.C. Panoiu, JETP Letters 65 (1997) 393.

[33] I.V. Mel'nikov, D. Mihalache, N.C. Panoiu, Optics Communications 181 (2000) 345.

[34] H. Leblond, F. Sanchez, Physical Review A 67 (2003) 013804.

[35] I.V. Mel'nikov, H. Leblond, F. Sanchez, D. Mihalache, IEEE Journal of Selected Topics in Quantum Electronics 10 (2004) 870.

[36] H. Leblond, F. Sanchez, I.V. Mel'nikov, D. Mihalache, Mathematics and Computers in Simulation 69 (2005) 378.

[37] H. Leblond, S.V. Sazonov, I.V. Mel'nikov, D. Mihalache, F. Sanchez, Physical Review A 74 (2006) 063815.

[38] H. Leblond, I.V. Mel'nikov, D. Mihalache, Physical Review A 78 (2008) 043802.

[39] H. Leblond, D. Mihalache, Physical Review A 79 (2009) 063835.

[40] H. Leblond, D. Mihalache, Journal of Optoelectronics and Advanced Materials 12 (2010) 1.

[41] H. Leblond, D. Mihalache, Romanian Reports in Physics 63 (2011) 1254.

[42] S.A. Skobelev, D.V. Kartashov, A.V. Kim, Physical Review Letters 99 (2007) 203902.

[43] Sh. Amiranashvili, A.G. Vladimirov, U. Bandelow, Physical Review A 77 (2008) 063821.

[44] R.K. Dodd, J.C. Eilbeck, J.D. Gibbon, H.C. Morris, Solitons and Nonlinear Wave Equations, Academic, London, 1982.

[45] M.J. Ablowitz, H. Segur, Solitons and the Inverse Scattering Transform, SIAM, Philadelphia, 1981.

[46] K. Konno, W. Kameyama, H. Sanuki, Journal of the Physical Society of Japan 37 (1974) 171.

[47] A.M. Kosevich, A.S. Kovalev, Solid State Communications 12 (1973) 763.

[48] E.V. Kazantseva, A.I. Maimistov, Physics Letters A 263 (1999) 434.

[49] E.V. Kazantseva, A.I. Maimistov, Quantum Electronics 30 (2000) 623.

[50] E.V. Kazantseva, A.I. Maimistov, B.A. Malomed, Optics Communications 188 (2001) 195.

[51] H. Leblond, Physical Review A 78 (2008) 013807.

[52] L. Bergé, S. Skupin, Physical Review Letters 100 (2008) 113902.

[53] H. Leblond, D. Kremer, D. Mihalache, Physical Review A 80 (2009) 053812.

[54] H. Leblond, D. Kremer, D. Mihalache, Physical Review A 81 (2010) 033824.

[55] H. Leblond, D. Mihalache, Physical Review A 81 (2010) 063815.

[56] N.N. Rosanov, V.V. Kozlov, S. Wabnitz, Physical Review A 81 (2010) 043815

[57] H. Leblond, D. Mihalache, Journal of Physics A: Mathematical and Theoretical 43 (2010) 375205.

[58] V.V. Kozlov, N.N. Rosanov, C. De Angelis, S. Wabnitz, Physical Review A 84 (2011) 023818.

[59] A. Pusch, J.M. Hamm, O. Hess, Physical Review A 84 (2011) 023827.

[60] E.D. Farnum, J.N. Kutz, Optics Letters 35 (2010) 3033.

[61] A.V. Kim, S.A. Skobolev, Physical Review A 83 (2011) 063832

[62] H. Leblond, H. Triki, F. Sanchez, D. Mihalache, Physical Review A 83 (2011) 063802.

[63] H. Leblond, H. Triki, F. Sanchez, D. Mihalache, Optics Communications 285 (2012) 356.

[64] H. Leblond, H. Triki, D. Mihalache, Physical Review A 84 (2011) 023833.

[65] H. Leblond, Journal of Physics B: Molecular and Optical Physics 41 (2008) 043001.

[66] R.W. Boyd, Nonlinear Optics, Third edition Academic, San Diego, 2007.

[67] H. Leblond, Journal of Physics A: Mathematical and General 34 (2001) 3109.

[68] Y.S. Kivshar, Optics Letters 16 (1991) 892.

[69] M. Wadati, Journal of the Physical Society of Japan 34 (1973) 1289.

[70] R. Hirota, Lecture Notes in Mathematics, vol. 515, Springer, Berlin, 1976, p. 40.

[71] S.M. Cox, P.C. Matthews, Journal of Computational Physics 176 (2002) 430. 